\title{
Technical note: A novel approach to estimate dry matter intake of lactating dairy cows through multiple on-cow accelerometers
}

\author{
N. A. Carpinelli, F. Rosa, R. C. B. Grazziotin, and J. S. Osorio* (1) \\ Dairy and Food Science Department, South Dakota State University, Brookings 57007
}

\section{ABSTRACT}

The objective of this study was to evaluate the feasibility of using multiple 3-dimensional accelerometers to estimated individual dry matter intake (DMI) of lactating dairy cows. Twenty-four Holstein cows in late lactation were assigned into 2 groups, a calibration group $(\mathrm{n}=12)$ and a validation group $(\mathrm{n}=12)$. All cows were fitted with 3 sensors that recorded 3 -dimensional acceleration (i.e., $\mathrm{x}, \mathrm{y}$, and $\mathrm{z}$ ) at 10-s intervals, 1 on the lateral side of the left hind leg and 2 attached directly to a halter over the nose and jaw area on the left side. Then, 3 accelerations were generated from each accelerometer (e.g., Leg-X, Leg-Y, and Leg-Z). Six new variables were created based on the change in acceleration in the nose and jaw accelerometers between 2 consecutive time points (e.g., LagJaw-X). For both groups (i.e., calibration and validation), cows were continuously video recorded while data on acceleration and intake of total mixed ration were collected for 10 consecutive days. Cows were fed once daily using an individual gate system, and individual refusals were recorded next day before morning feeding. Cows were fed a common lactating cow diet (17.9\% crude protein; $1.70 \mathrm{Mcal} / \mathrm{kg}$ of dry matter). In the calibration group, individual eating bouts were obtained based on video recordings and merged with the corresponding accelerometer data. Then, a stepwise regression analysis was conducted using the REG procedure of SAS (SAS Institute, Cary, NC) to determine the ranges in acceleration that accounted for the highest variation in DMI (highest $\mathrm{R}^{2}$ ) in each acceleration variable. All 32,767 potential acceleration combinations were tested in the validation group using the acceleration ranges predetermined in the calibration group. The CORR procedure of SAS was used to test the Pearson correlation coefficient (r) between the type of DMI [i.e., based on accelerations $\left(D M I_{\text {accel }}\right)$ or actual DMI $\left.\left(D M I_{\text {actual }}\right)\right]$. The MIXED procedure of SAS was used to perform

Received February 24, 2019.

Accepted July 8, 2019.

*Corresponding author: Johan.Osorio@sdstate.edu a repeated-measures analysis with type $\left(D M I_{\text {accel }}\right.$ vs. $\left.D M I_{\text {actual }}\right)$, day, and their interaction $(\mathrm{T} \times \mathrm{D})$ in the model. From this analysis, 8 candidate acceleration models were selected based on high $\mathrm{r}$ and similarity $(P$ $>0.15)$ in terms of $\mathrm{T}$ and $\mathrm{T} \times \mathrm{D}$ between $D M I_{\text {accel }}$ and $D M I_{\text {actual }}$. A simulated effect on $D M I_{\text {actual }}$ was artificially created in the validation group by dividing this group $(\mathrm{n}=12)$ into high and low intake cows $(\mathrm{n}=6$ /group; DMI of 24.1 vs. $18.7 \mathrm{~kg} / \mathrm{d}$ ), and the candidate models were tested to determine whether they were sensitive enough to detect this effect. From these candidate models, AEN (Leg-X + Jaw-Z + LagJaw-Z) showed a weak correlation $(\mathrm{r}=0.36)$ between $D M I_{\text {accel }}$ and $D M I_{\text {actual }}$, but $D M I_{\text {accel }}$ and $D M I_{\text {actual }}$ were highly similar (21.2 vs. $21.4 \mathrm{~kg} / \mathrm{d}$ of DMI). In addition, this was the only model that could detect the simulated effect on $D M I_{\text {actual }}(22.1$ vs. $20.3 \mathrm{~kg} / \mathrm{d}$ of DMI) in the validation group. The fact that the simulated effect on $D M I_{\text {actual }}$ was detected based only on accelerations is highly significant, and models such as AEN could be substantially improved if they were derived from a greater sample size and included different physiological stages in dairy cows.

Key words: accelerometer, feed intake, sensor technology

\section{Technical Note}

The typical dairy farm worldwide has changed over the years and the number of cows per dairy farm has increased, making it more difficult for producers to devote adequate time to each animal (Bikker et al., 2014). This trend presents a tremendous challenge to dairy farm management and can be exacerbated if adequate protocols are not followed and records are not consistent or up to date (Wenz, 2018). For these reasons, rapid adoption of precision dairy farm technologies has occurred in recent years, allowing farmers to monitor cows individually, and subsequently providing valuable information to support decision-making and improve farm productivity and profitability (van Asseldonk et al., 1999).

Dry matter intake is a fundamental parameter in dairy nutrition research because it allows estimation of 
overall supply of nutrients, especially those being evaluated during research experiments. Dry matter intake is essential to correlated health status during stress periods such as the transition period in dairy cows, and it is fundamental in calculating the overall milk efficiency in dairy cows (i.e., $\mathrm{kg}$ of milk/ $\mathrm{kg}$ of DMI). The DMI in lactating cows can be affected by physical and chemical characteristics (e.g., DM and NDF content) of dietary ingredients and their interactions (Allen, 2000). These factors and their interactions make it challenging for any attached or nonattached sensor system to estimate actual DMI in a TMR format in dairy cows. In contrast, the aforementioned factors and their interactions are less problematic when DMI is measured by TMR disappearance (i.e., TMR offered - TMR refused) in research experiments through a gate system or automatic feeder system that can continuously record intake on small dairy farms (Schirmann et al., 2011). However, these methods of recording DMI are not feasible for large-scale dairy farm operations.

Sensor system technology has been used for estimated behaviors such as lying behavior (Ledgerwood et al., 2010; Rodriguez-Jimenez et al., 2018), rumination (Zehner et al., 2017; Grinter et al., 2019; Zambelis et al., 2019) and feeding behavior (Bikker et al., 2014; Büchel and Sundrum, 2014; Mattachini et al., 2016). Individual feeding behavior collected in real time through sensor systems is a valuable tool for dairy farmers and is commonly associated with health status in the herd. Although this information helps farmers monitor cows at risk of developing a disease, most sensor systems currently only estimate feeding behavior as a percentage of time during a 24-h period (Bikker et al., 2014). Predicting actual DMI in real time through an accelerometer sensor system would be an additional tool for dairy farmers that could be used to monitor herd health and provide an estimate of milk efficiency on an individual cow basis. Therefore, the objective of this study was to evaluate the feasibility of using a 3-dimensional accelerometer sensor system for the estimation of individual feed intake of lactating dairy cows.

All the protocols for this study (protocol no. 17-082E) were approved by the South Dakota State University Institutional Animal Care and Use Committee (IACUC). The study was conducted between October and November 2017 at the Dairy Research and Training Facility in Brookings.

Twenty-four Holstein cows in late lactation (266 $\pm 87 \mathrm{DIM}$ ) with an average parity of $2.6 \pm 0.7$ were housed in freestall barns and divided into 2 groups, a calibration group $(\mathrm{n}=12)$ and a validation group ( $\mathrm{n}$ $=12$ ). Cows were trained to use the Calan gate feeding system (American Calan Inc., Northwood, NH) and were adapted to the research pens for $7 \mathrm{~d}$ before data collection. For both groups, data on acceleration, intakes of TMR, and behavior through continuous video recordings were collected for 10 consecutive days. All cows were fitted with 3 sensors (Hobo Pendant G Data Logger, Onset Computer Corp., Bourne, MA) that recorded 3-dimensional acceleration in 3 axes (i.e., $\mathrm{x}$, $y$, and $z$ ) at 10-s intervals, 1 on the lateral side of the left hind leg and 2 attached directly to a halter over the nose and on the jaw area on the left side. Each cow wore the halter throughout the experiment (Supplemental Figure S1; https://doi.org/10.3168/jds.2019 -16537). Cows were recorded continuously throughout the $10 \mathrm{~d}$ of data collection with a surveillance system of a 960P wireless network video recorder equipped with 4 cameras with motion sensors and night vision (cat no. ZSWNVK-A41000, ZOSI Tech, Hong Kong). Cameras were positioned with the aim of completely covering all individual feeding gates. Two trained individuals watched the video continuously and recorded the time when an eating behavior event began (when a cow entered her assigned gate and remained eating, standing idle, or ruminating). The individuals had an interobserver reliability of $75 \%$ and an intraobserver reliability of $95 \%$ for total eating bouts for a subset of the data set $(\mathrm{n}=12$ cows for $10 \mathrm{~h})$. Agreement was calculated as correlations in Excel (Microsoft Corp., Redmond, WA). Individual daily feed intake of the cows was recorded daily by feeding cows once daily at $0630 \mathrm{~h}$ and subtracting the manually weighed refusals the next day before the morning feeding. Cows were fed a common lactating cow diet $(17.9 \% \mathrm{CP} ; 1.70 \mathrm{Mcal} /$ $\mathrm{kg}$ of DM) throughout the experiment (Supplemental Table S1; https://doi.org/10.3168/jds.2019-16537). Throughout the study, TMR samples were taken daily for determination of DM and nutrient composition. Cows were milked twice a day in a double- 8 parallel parlor. Individual cow BW and BCS were recorded at 1,5 , and $10 \mathrm{~d}$ of the trial.

In the validation group, the video recordings were examined after the trial and provided a continuous account of all Calan gates throughout the day (24-h period), allowing eating bouts to be determined for each cow. Eating bouts were classified throughout the day as each minute the cow remained eating, standing idle, or ruminating in the assigned Calan gate with the door open. The latter was the first step in a logical progression to determine the best model to estimate DMI from the acceleration data described above (Figure 1). The accelerometer data from the calibration group were merged with the corresponding eating bouts, and all accelerometer data when cows were not in the Calan gate (not eating behavior) were removed. Because cows lower their nose to a feed bunk table level before every eating bout, we deemed this behavior important 
to estimate intake. Therefore, we accounted for this behavior through the accelerometer data by measuring the change in acceleration between 2 consecutive time points in the jaw and nose accelerometers, using a lag-time approach similar to that used by Ledgerwood et al. (2010). Based on this, we generated 6 new "Lag" variables (e.g., difference in x-axis nose, where LagNose-X $=$ Nose-X-Time $_{1}-$ Nose-X-Time $_{0}$ ) derived from jaw and nose accelerations. To facilitate data handling of these new variables, the minimum of value of each variable was added to all values within the same variable to make all delta $g$ forces positive. All variables were analyzed with PROC MEANS of SAS (SAS Institute Inc., Cary, NC) to determine the minimum, quartile (Q)1, median (Q2), quartile 3 (Q3), and maximum values with the aim of dividing each data variable into 4 segments. The number of times during the day a cow performed an acceleration within each segment (as defined by quartiles) was counted as an event. Then, PROC REG was used to perform a stepwise regression in each acceleration variable by regressing the number of events/24 $\mathrm{h}$ for each segment in a given acceleration against the actual DMI (DMI $\left.I_{\text {actual }}\right)$ during the same 24 $\mathrm{h}$ for the respective cow. This analysis was repeated in a backward elimination fashion, by eliminating the segments (or regions) with low $\mathrm{R}^{2}$ and leaving only the segments of each acceleration with high $\mathrm{R}^{2}$ that accounted for a lot of the variation observed in DMI (Supplemental Figure S2; https://doi.org/10.3168/jds .2019-16537). For instance, when considering all the LagNose-X (i.e., Nose-X-Time ${ }_{1}-$ Nose-X-Time $_{0}$ ) data, we observed an $\mathrm{R}^{2}$ of 0.34 (data not shown); however, when the range of these data was narrowed to a lower and upper bound of 0.5 and 0.52 , respectively, the highest $\mathrm{R}^{2}$ was obtained, accounting for $44 \%$ of the variation in $D M I_{\text {actual }}$ (Table 1 ). Therefore, the curated ranges of each acceleration presented in Table 1 can be considered the movements or behaviors expressed by cows while eating and captured within these narrowed segments in each acceleration. Although the final $\mathrm{R}^{2}$ for each acceleration was not particularly high $(<44 \%)$, it is plausible that a specific combination of these accelerations will return a higher $\mathrm{R}^{2}$ or a better adjustment to estimate DMI in dairy cows.

The determination of the highest $\mathrm{R}^{2}$ segments in each acceleration variable allowed the determination of the best candidate models to estimate DMI in the calibration group using the standard regression model:

$$
D M I_{\text {calibration }}=B_{0}+B_{1} \times X_{c}+e_{c}
$$

where $D M I_{\text {calibration }}$ is the DMI estimate based on the number of events derived from multiple accelerations in the calibration group; $B_{0}$ is the intercept in the model

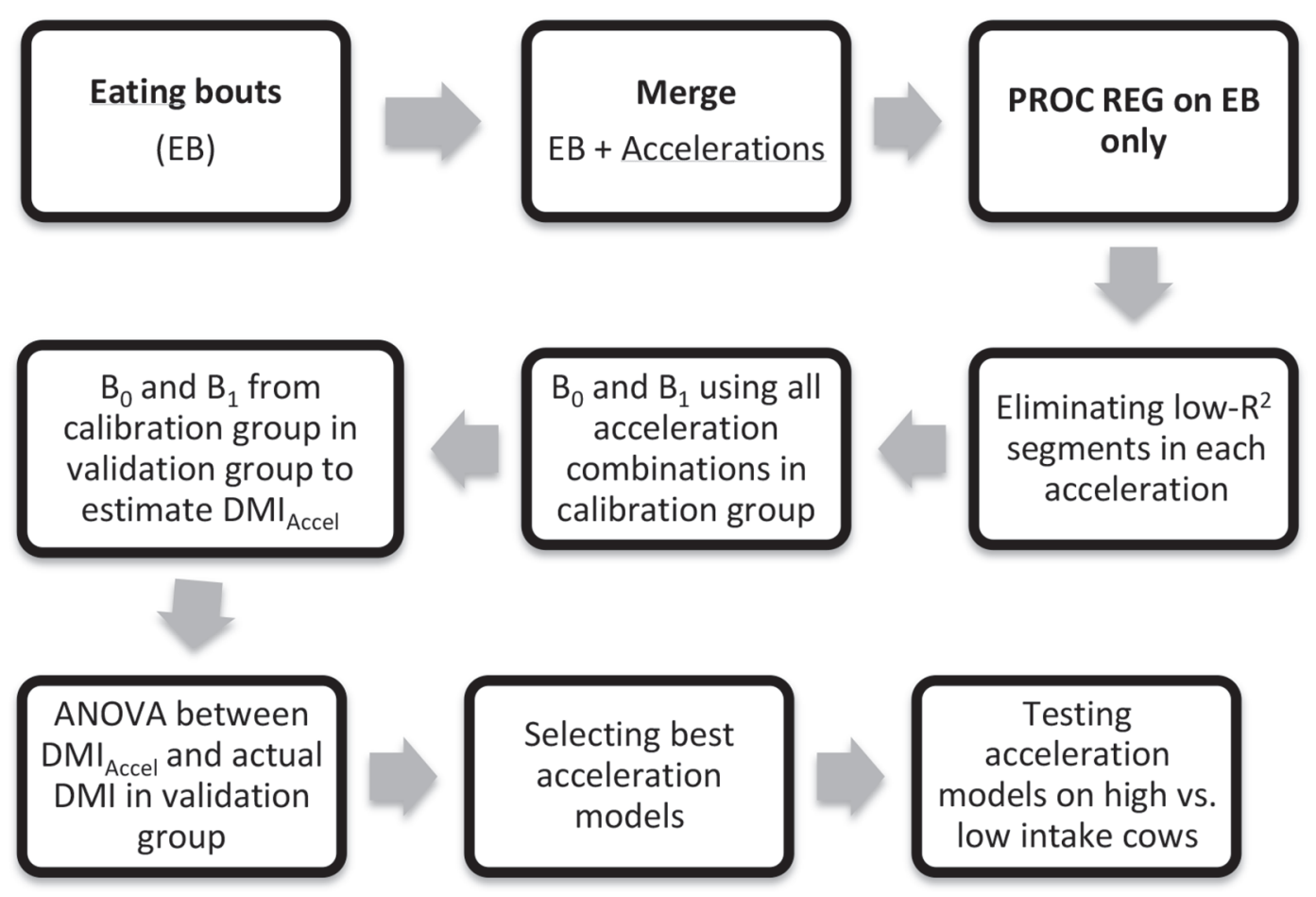

Figure 1. Logical progression on the development and testing of candidate acceleration models to estimate daily intake using accelerometer sensor data in lactating dairy cows. $\mathrm{B}_{0}$ (intercept) and $\mathrm{B}_{1}$ (slope) were derived from regression models. 
to determine the lowest estimable DMI; $B_{1}$ is the slope of the model and rate of increment in DMI by unit of events derived from multiple accelerations; $X_{c}$ is the cumulative number of events $/ 24 \mathrm{~h}$ based on a specific combination of accelerations; and $e_{c}$ is the residual error of the model.

The next step in the analysis (Figure 1) was to estimate $D M I_{\text {accel }}$ in the validation group by utilizing the coefficients (i.e., $B_{0}$ and $B_{1}$ ) determined in the calibration group and the cumulative number of events $/ 24 \mathrm{~h}$ based on accelerations in the validation group. It is important to emphasize that although $D M I_{\text {calibration }}$ estimated in the calibration group was done on accelerations during eating bouts only, $D M I_{\text {accel }}$ estimated in the validation group was done on accelerations throughout the day. This represents a substantial challenge because of a significant increase in noise (e.g., cow acceleration while resting, walking, standing idly) in validation group data, which can consequently affect the accuracy of the $D M I_{\text {accel }}$ estimation. However, this is by design because this technology aims to be able to estimate $D M I_{\text {accel }}$ during a 24 -h period based only on accelerations. The $D M I_{\text {accel }}$ in the validation group was estimated using the following model:

$$
D M I_{a c c e l}=B_{0 c}+B_{1 c} \times X_{v}+e_{v}
$$

where $D M I_{\text {accel }}$ is the DMI estimate in the validation group based on the number of events derived from multiple accelerations; $B_{0 c}$ is the intercept in the model determined from the calibration group; $B_{1 c}$ is the slope of the model determined from the calibration group; $X_{v}$ is the cumulative number of events/24 h based on a specific combination of accelerations in the validation group; and $e_{v}$ is the residual error of the model in the validation group.

All 32,767 potential combinations of the 15 acceleration variables (Table 1) were tested, and only 921 acceleration combinations were deemed significant for further evaluation because each contained at least $80 \%$ of all potential data. Each of the 15 acceleration parameters was abbreviated to an alphabetic letter for simplification purposes (Table 1). To establish a direct comparison between $D M I_{\text {accel }}$ and $D M I_{\text {actual }}$ recorded in the validation group, a repeated-measures analysis was performed using PROC MIXED of SAS, where the model included type (i.e., $D M I_{\text {accel }}$ or $D M I_{\text {actual }}$ ), day, and their interaction as fixed effects, and cow was nested within type as the random effect. Additionally, a correlation analysis using PROC CORR was carried out between $D M I_{a c c e l}$ and $D M I_{\text {actual }}$ in the validation group. Then, results from the repeated-measures and correlation analyses were used as tools to generate cri-
Table 1. Acceleration variables with their respective abbreviation and $\mathrm{R}^{2}$ associated with $D M I_{\text {actual }}$ on the 3 axes (i.e., $\mathrm{X}, \mathrm{Y}$, and $\mathrm{Z}$ ) for each accelerometer in the leg, nose, and left jaw

\begin{tabular}{lllll}
\hline & & \multicolumn{2}{c}{ Bound $^{1}$} \\
Variable & & \\
& Abbreviation & Lower & Upper & $\mathrm{R}^{2}$ \\
\hline Accelerations & & & & \\
Leg & & & & \\
X & $\mathrm{A}$ & 3.18 & 3.28 & 0.20 \\
Z & $\mathrm{B}$ & 2.04 & 2.4 & 0.20 \\
Y & $\mathrm{C}$ & 3.18 & 3.57 & 0.30 \\
Jaw & & & & \\
X & $\mathrm{D}$ & 2.54 & 2.76 & 0.26 \\
Z & $\mathrm{E}$ & 2.83 & 3.11 & 0.21 \\
Y & $\mathrm{F}$ & 2.19 & 2.57 & 0.27 \\
Nose & & & & \\
X & $\mathrm{G}$ & 2.94 & 2.96 & 0.24 \\
Z & $\mathrm{H}$ & 3.58 & 3.86 & 0.27 \\
Y & $\mathrm{I}$ & 2.12 & 2.14 & 0.26 \\
Change in acceleration & & & & \\
Lag Nose & & & & \\
X & & & & \\
Z & $\mathrm{J}$ & 0.50 & 0.52 & 0.44 \\
Y & $\mathrm{K}$ & 0.50 & 0.53 & 0.35 \\
Lag Jaw & $\mathrm{L}$ & 0.50 & 0.52 & 0.35 \\
X & & & & \\
Z & $\mathrm{M}$ & 0.44 & 0.50 & 0.38 \\
Y & $\mathrm{N}$ & 0.50 & 0.52 & 0.38 \\
I & $\mathrm{O}$ & 0.51 & 0.57 & 0.35 \\
\hline
\end{tabular}

${ }^{1}$ Final ranges in each acceleration variable that return the highest $\mathrm{R}^{2}$ associated with the $D M I_{\text {actual }}$.

${ }^{2}$ For each nose and jaw acceleration, a change in acceleration was determined as the difference of $\mathrm{Time}_{1}-\mathrm{Time}_{0}$ [e.g., LagNose-X $=$ $\left(\right.$ Nose- $\mathrm{X}_{1}-$ Nose- $\left.\mathrm{X}_{0}\right)$ ].

teria to select the best candidates of acceleration combinations that could explain the variation in $D M I_{\text {actual }}$ and generate a $D M I_{\text {accel }}$ that is similar $(P>0.15)$ to $D M I_{\text {actual }}$. Within the 921 acceleration combinations mentioned above, the KL (LagNose-Z + LagNose-X) and KN (LagNose-Z + LagJaw-Z) acceleration models had the highest correlation $(\mathrm{r}=0.42$ and $\mathrm{r}=0.39 ; P<$ 0.01 , respectively), whereas CEM (Leg-X + Jaw-X + LagNose-Y) and AELM (Jaw-X + Jaw-Z + Jaw-Y + LagNose-Z) produced the closest $(P>0.99) D M I_{\text {accel }}$ to $D M I_{\text {actual }}$ (Table 2). It is clear that the high correlation between $D M I_{\text {accel }}$ and $D M I_{\text {actual }}$ observed in models $\mathrm{KL}$ and $\mathrm{KN}$ did not translate into an accurate prediction of $D M I_{\text {actual }}$ because they overestimated DMI by 7.9 and $14.8 \mathrm{~kg} / \mathrm{d}$, respectively (Figure 2). In contrast, models with high similarity between $D M I_{\text {accel }}$ and $D M I_{\text {actual }}$, such as CEM and AELM, had lower r (-0.11 and 0.22, respectively); in the case of CEM, $\mathrm{r}$ was not significant $(P=0.24)$. The latter diminishes the robustness of these models if they are to be used across several conditions such as early lactation, parity, by weight, and so on. Models that produced a high similarity (or high $P$-value) in terms of the interaction $\mathrm{T} \times \mathrm{D}$ will likely be able to produce a $D M I_{\text {accel }}$ that closely follows the changes over time in $D M I_{\text {actual }}$. Based on the latter, 
we selected GI (Nose-X + Nose-Y) and EIK (Jaw-Z + Nose-Y + LagNose-Z) because they had the highest similarity on $\mathrm{T} \times \mathrm{D}(P \geq 0.78)$. However, the GI and EIK models showed negative correlations $(\mathrm{r}=-0.33$ and $\mathrm{r}=-0.26 ; P<0.01$, respectively) between $D M I_{\text {accel }}$ and $D M I_{\text {actual }}$, which could be interpreted as cows expressing more of these types of acceleration patterns (i.e., GI and EIK), will be biased to have lower $\mathrm{DMI}_{\text {accel }}$ than $\mathrm{DMI}_{\text {actual }}$. This will render these models less accurate for low-intake behaviors such as those commonly observed during the onset of a disease or around calving (Rodriguez-Jimenez et al., 2018). Because of the limitations on the models mentioned above, another set of models was selected based on $\mathrm{r} \geq 0.3$ and high similarity $(P \geq 0.90)$ based on type (e.g., $D M I_{\text {accel }}$ vs. $\left.D M I_{\text {actual }}\right)$. These 2 criteria were used to select the models AEN (Leg-X + Jaw-Z + LagJaw-Z) and DEFM (Jaw-X + Jaw-Z + Jaw-Y + LagJaw-Y) as intermediate models. These intermediate models were able to predict a DMI (21.2 and $21.3 \mathrm{~kg} / \mathrm{d}$ for AEN and DEFM, respectively) that was highly similar to $D M I_{\text {actual }}$, and a comparable r (0.36 and 0.30 for AEN and DEFM, respectively) to those acceleration models (i.e., KL and $\mathrm{KN}$ ) with the strongest correlations. Therefore, the AEN and DEFM models might more robust to predict intake and adjust for changes in cows' behavior.

To test the robustness of the candidate models selected in this study (Table 2), we devised an approach where we simulated an effect in $D M I_{\text {actual }}$ in the valida- tion group. This simulation was carried out by dividing cows with high and low $D M I_{\text {actual }}$ into high $(\mathrm{n}=6)$ and low $(\mathrm{n}=6)$ groups in the validation group. Then, the candidate models (Table 2) were used to estimate $D M I_{a c c e l}$ in the high and low groups of cows and confirm whether the simulated effect in $D M I_{\text {actual }}$ was detected in the $D M I_{\text {accel }}$ derived from those candidate models. This simulation exercise indicated whether these candidate models were robust enough to predict a $D M I_{\text {accel }}$ that could reproduce the simulated effect in $D M I_{\text {actual }}$. As expected, an effect in $D M I_{\text {actual }}$ was observed such that the high-intake group had a greater $(P<0.01)$ DMI $(\sim 5 \mathrm{~kg} / \mathrm{d}$ more) than the low-intake group (Table 3 ). Interestingly, only the AEN model was able to detect a similar effect between the high- and low-intake groups, where the high group had $\sim 1.8 \mathrm{~kg} /$ d greater $(P<0.01)$ $D M I_{\text {accel }}$ than the low group. The EIK and GI models presented a significant effect $(P=0.04)$ and a trend $(P=0.07)$ for a difference between the high- and lowintake groups in $D M I_{\text {accel }}$, respectively; however, both of these models estimated greater $D M I_{\text {accel }}$ in the low group than in the high group. The inability to detect changes in $D M I_{\text {accel }}$ between the low- and high-intake groups by models with a strong correlation between $D M I_{\text {accel }}$ and $D M I_{\text {actual }}$ (i.e., KL and KN) or high similarity between $D M I_{\text {accel }}$ and $D M I_{\text {actual }}$ (i.e., CEM and AELM) underscores the need to improve robustness in this accelerometer sensor approach to accurately estimate DMI. In the case of the AEN model, even though it was able

Table 2. Eight selected candidate models based on their correlation and similarity between $D M I_{\text {accel }}$ (DMI derived from each acceleration combination) and actual DMI in the validation group

\begin{tabular}{|c|c|c|c|c|c|c|c|c|}
\hline Acceleration model $^{1}$ & \multicolumn{2}{|c|}{ Intake $^{2}$} & SEM & \multicolumn{3}{|c|}{$P$-value ${ }^{3}$} & \multicolumn{2}{|c|}{ Correlation $^{4}$} \\
\hline \multicolumn{9}{|l|}{ Strongest correlation } \\
\hline $\mathrm{KN}$ & 29.3 & 21.4 & 0.9 & $<0.01$ & 0.01 & 0.19 & 0.39 & $<0.01$ \\
\hline \multicolumn{9}{|l|}{ Highly similar ${ }^{5}$} \\
\hline CEM & 21.4 & 21.4 & 0.8 & 0.99 & 0.01 & 0.09 & -0.11 & 0.24 \\
\hline GI & 21.9 & 21.4 & 1.0 & 0.72 & 0.21 & 0.90 & -0.33 & $<0.01$ \\
\hline EIK & 21.9 & 21.4 & 0.9 & 0.66 & 0.20 & 0.78 & -0.26 & $<0.01$ \\
\hline \multicolumn{9}{|l|}{ Intermediate models $^{7}$} \\
\hline $\mathrm{AEN}$ & 21.2 & 21.4 & 0.8 & 0.90 & 0.38 & 0.10 & 0.36 & $<0.01$ \\
\hline DEFM & 21.3 & 21.4 & 0.8 & 0.94 & 0.14 & 0.12 & 0.30 & $<0.01$ \\
\hline
\end{tabular}

${ }^{1}$ Accelerations on the 3 axes $(\mathrm{X}, \mathrm{Y}$, and $\mathrm{Z}$ ) located in the leg, jaw, and nose as well as the LagNose and LagJaw variables $[$ e.g., LagNose-X $=$ $\left(\right.$ Nose-X-Time $_{1}-$ Nose-X-Time $\left._{0}\right)$ ] were used to determine the acceleration models, defined as follows: KL = LagNose-Z + LagNose-X; KN $=$ LagNose-Z + LagJaw-Z; GI = Nose-X + Nose-Y; EIK = Jaw-Z + Nose-Y + LagNose-Z; CEM = Leg-Y + Jaw-Z + LagNose-Y; AELM = Leg-X + Jaw-Z + LagNose-X + LagNose-Y; AEN = Leg-X + Jaw-Z + LagJaw-Z; DEFM = Jaw-X + Jaw-Z + Jaw-Y + LagJaw-Y.

${ }^{2}$ Overall estimated DMI derived from accelerations (accel; i.e., $D M I_{\text {accel }}$ ) and actual DMI.

${ }^{3} P$-values associated with type $\left(\mathrm{T} ; D M I_{a c c e l}\right.$ and actual DMI), day $(\mathrm{D})$, and their interactions $(\mathrm{T} \times \mathrm{D})$.

${ }^{4}$ Correlation coefficient and $P$-value associated between $D M I_{\text {accel }}$ and actual DMI.

${ }^{5}$ Acceleration models with the highest similarity $(P \geq 0.90)$ in terms of $\mathrm{T}$.

${ }^{6}$ Acceleration models with the highest similarity $(P \geq 0.78)$ in terms of $\mathrm{T} \times \mathrm{D}$.

${ }^{7}$ Intermediate acceleration models classified by having an $\mathrm{r} \geq 0.30$ and a high similarity $(P \geq 0.90)$ in terms of $\mathrm{T}$. 


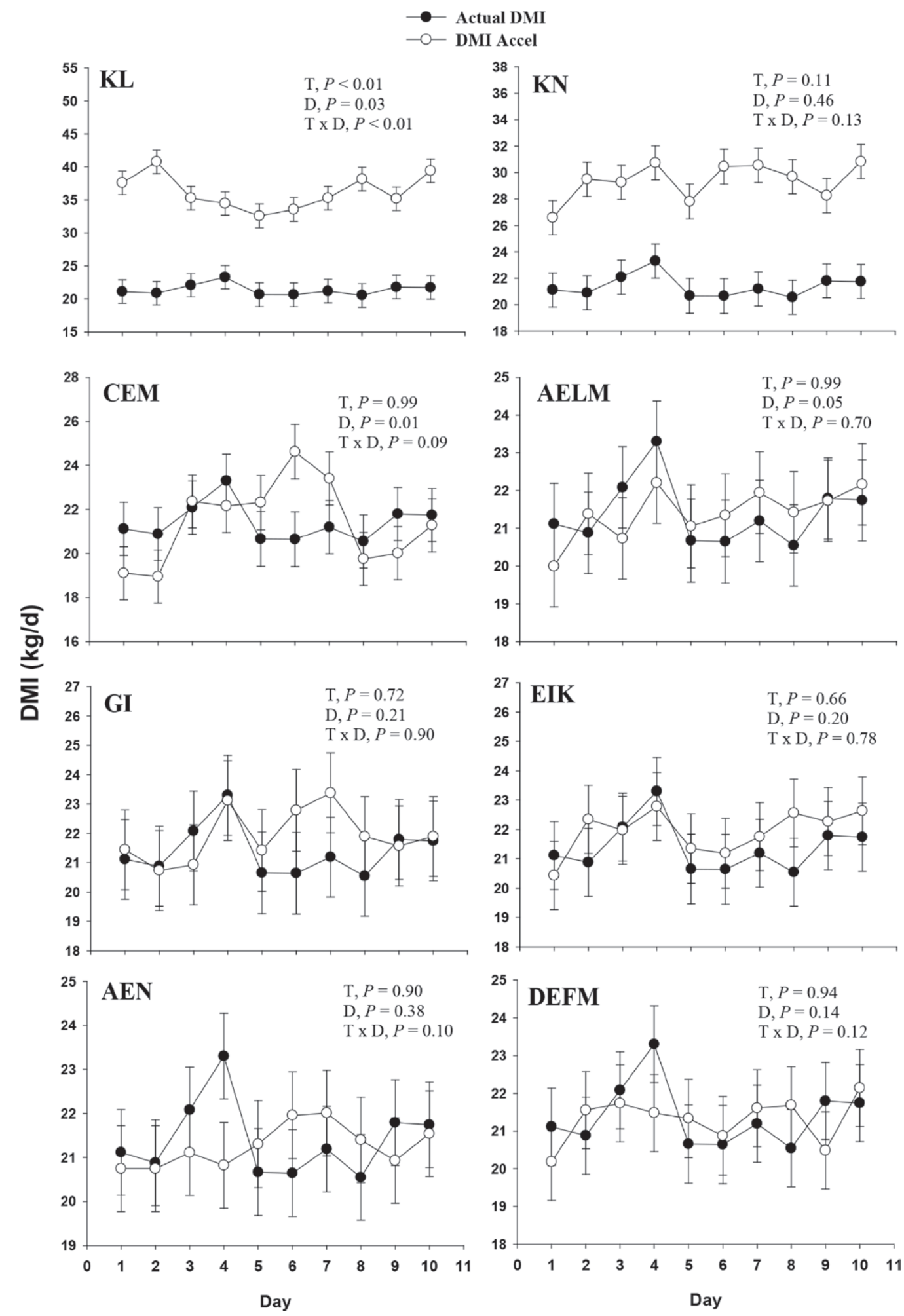

Figure 2. Comparison over time between actual DMI and $D M I_{\text {accel }}$ derived from selected acceleration models based on correlation ( $\mathrm{r} \geq 0.39$; $\mathrm{KL}$ and KN), similarity in terms of type (T, $P=0.99$; CEM and AELM), interaction between type and day $(\mathrm{T} \times \mathrm{D}, P \geq 0.78 ; \mathrm{GI}$ and EIK), and intermediate models $(\mathrm{r} \geq 0.30 ; \mathrm{T}, P \geq 0.90$; AEN and DEFM). Accelerations on the 3 axes (X, Y, and $\mathrm{Z})$ from the leg, jaw, and nose accelerometers, as well as the LagNose and LagJaw variables [e.g., LagNose-X $=\left(\right.$ Nose-X-Time ${ }_{1}-$ Nose-X-Time $\left._{0}\right)$ ] were used to determine KL $=$ LagNose-Z + LagNose-X; KN = LagNose-Z + LagJaw-Z; GI = Nose-X + Nose-Y; EIK = Jaw-Z + Nose-Y + LagNose-Z; CEM = LegY + JawZ + LagNoseY; AELM = LegX + JawZ + LagNoseX + LagNoseY; AEN = Leg-X + Jaw-Z + LagJaw-Z; DEFM = Jaw-X + Jaw-Z + Jaw-Y + LagJaw-Y. Values are given as means; error bars represent SE. 
Table 3. The simulated effect in DMI in the validation group to generate high- and low-intake groups of cows, and tested across all acceleration models

\begin{tabular}{|c|c|c|c|c|c|c|}
\hline \multirow[b]{2}{*}{ Acceleration model $^{1}$} & \multicolumn{2}{|c|}{ Intake $^{2}$} & \multirow[b]{2}{*}{ SEM } & \multicolumn{3}{|c|}{$P$-value ${ }^{3}$} \\
\hline & High & Low & & $\mathrm{T}$ & $\mathrm{D}$ & $\mathrm{T} \times \mathrm{D}$ \\
\hline Actual DMI & 24.1 & 18.7 & 0.9 & $<0.01$ & 0.04 & 0.01 \\
\hline \multicolumn{7}{|l|}{ Strongest correlation } \\
\hline KL & 38.3 & 34.2 & 2.0 & 0.17 & 0.02 & 0.70 \\
\hline $\mathrm{KN}$ & 41.3 & 38.6 & 2.5 & 0.48 & 0.37 & 0.09 \\
\hline \multicolumn{7}{|l|}{ Highly similar ${ }^{4}$} \\
\hline CEM & 20.9 & 21.9 & 0.6 & 0.28 & 0.01 & 0.17 \\
\hline AELM & 22.1 & 20.7 & 0.7 & 0.19 & 0.42 & 0.23 \\
\hline \multicolumn{7}{|c|}{ Highly similar interaction ${ }^{5}$} \\
\hline GI & 20.1 & 23.7 & 1.2 & 0.07 & 0.77 & 0.69 \\
\hline EIK & 20.6 & 23.3 & 0.8 & 0.04 & 0.77 & 0.23 \\
\hline \multicolumn{7}{|l|}{ Intermediate models ${ }^{6}$} \\
\hline AEN & 22.1 & 20.3 & 0.3 & $<0.01$ & 0.78 & 0.17 \\
\hline DEFM & 21.8 & 20.8 & 0.8 & 0.40 & 0.35 & 0.81 \\
\hline
\end{tabular}

${ }^{1}$ Accelerations on the 3 axes (X, Y, and Z) from the leg, jaw, and nose accelerometers as well as the LagNose and LagJaw variables [e.g., LagNose-X $=\left(\right.$ Nose-X-Time $_{1}-$ Nose-X-Time $\left._{0}\right)$ ] were used to determine the acceleration models, defined as $\mathrm{KL}=$ LagNose-Z + LagNose-X; KN = LagNose-Z + LagJaw-Z; GI = Nose-X + Nose-Y; EIK = Jaw-Z + Nose-Y + LagNose-Z; CEM = LegY + JawZ + LagNoseY; AELM = LegX + JawZ + LagNoseX + LagNoseY; AEN = Leg-X + Jaw-Z + LagJaw-Z; DEFM = Jaw-X + Jaw-Z + Jaw-Y + LagJaw-Y.

${ }^{2}$ Actual DMI and $D M I_{\text {accel }}$ derived from acceleration combinations in high- and low-intake cows in the validation group.

${ }^{3} P$-values associated with type $\left(\mathrm{T} ; D M I_{\text {accel }}\right.$ and actual DMI), day $(\mathrm{D})$, and their interactions $(\mathrm{T} \times \mathrm{D})$.

${ }^{4}$ Acceleration models with the highest similarity $(P \geq 0.90)$ in terms of $\mathrm{T}$.

${ }^{5}$ Acceleration models with the highest similarity $(P \geq 0.78)$ in terms of $\mathrm{T} \times \mathrm{D}$.

${ }^{6}$ Intermediate acceleration models classified by having $\mathrm{r} \geq 0.30$ and a high similarity $(P \geq 0.90)$ in terms of $\mathrm{T}$.

to detect the expected differences between high- and low-intake cows, the difference returned in $D M I_{\text {accel }}$ was lower than that in $D M I_{\text {actual }}(1.8$ vs $5.4 \mathrm{~kg} / \mathrm{d}$; Figure 3). Also, the latter implies that this model, as is, will require that physiological or nutritional effects in DMI be $>5 \mathrm{~kg} / \mathrm{d}$ to be detected by this model, which is less realistic. However, the fact that we were able to detect this difference based only on accelerations is highly significant, especially because this analysis of high- versus low-intake cows in the validation group was generated with a limited number of biological replicates $(n=6 /$ group). Therefore, it is conceivable that the accuracy of a model such as AEN could be substantially improved with a greater sample size and including different physiological stages and stages of lactation.

From a management or research perspective, individual daily intake is a fundamental piece of information to either make appropriate decisions at the commercial farm level or to determine the impact of a nutritional or physiological effect. To our knowledge, this is the first attempt to estimate actual DMI in dairy cows based on a multiple on-cow accelerometer sensor system. In recent years, commercially available accelerometer and alternative sensor systems (Beauchemin, 2018) have been rapidly adopted by the dairy industry, and they have proven useful in providing current eating behavior data to dairy farmers, allowing farmers, in turn, to efficiently monitor a greater number of cows and flag those animals with abnormal eating behaviors. The multiple accelerometer approach and derived acceleration models proposed here suggest that cows have specific movements highly correlated with intake. However, the acceleration models generated here reached only moderate correlations. It is conceivable that by increasing the sample size, more frequent DMI measurements (e.g., hourly DMI) and a wider DMI range including low-intake cows (i.e., peripartal dairy cows) would substantially improve the robustness of these acceleration models to predict intake. Predicting intake in dairy cows is a challenging endeavor, and this was reflected in the amount of noise observed in this study based on accelerations alone. However, we envision that accurate accelerometer-based algorithms to estimate intake will allow dairy farmers to not only monitor abnormal eating behavior in dairy cows but also determine milk efficiency.

\section{ACKNOWLEDGMENTS}

This work was supported by the South Dakota Agricultural Experiment Station (Brookings) under project no. SD00H612-16. 


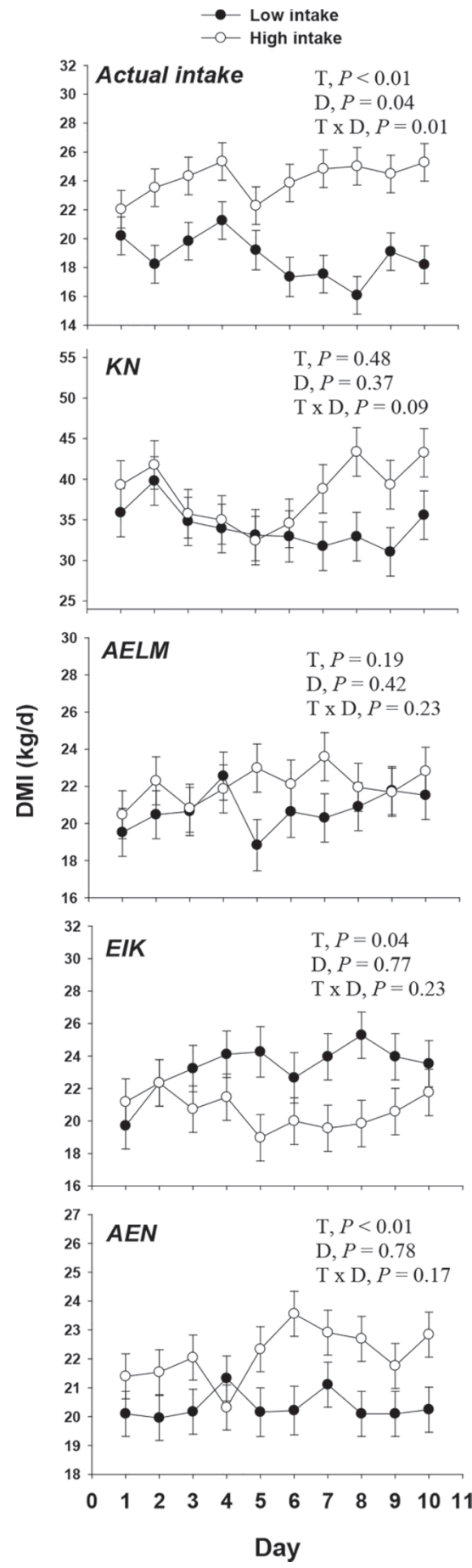

Figure 3. Comparison over time between high- and low-intake cows in the validation group as actual intake and $D M I_{\text {accel }}$ derived from selected acceleration models based on correlation ( $\mathrm{r} \geq 0.39 ; \mathrm{KN})$, similarity in terms of type (T, $P=0.99$; AELM), interaction between type and day $(\mathrm{T} \times \mathrm{D}, P \geq 0.78 ; \mathrm{EIK})$, and intermediate models $(\mathrm{r}$ $\geq 0.30 ; \mathrm{T}, P \geq 0.90 ; \mathrm{AEN})$. Accelerations on the 3 axes (X, Y, and $\bar{Z})$ from the leg, jaw, and nose accelerometers as well as the LagNose and LagJaw variables [e.g., LagNose-X $=\left(\right.$ Nose-X-Time $_{1}-$ Nose-X- $^{-}$ Time $_{0}$ )] were used to determine KN = LagNose-Z + LagJaw-Z; EIK = Jaw-Z + Nose-Y + LagNose-Z; AELM = LegX + JawZ + LagNoseX + LagNoseY; AEN = Leg-X + Jaw-Z + LagJaw-Z. Values are given as means; error bars represent $\mathrm{SE}$.

\section{REFERENCES}

Allen, M. S. 2000. Effects of diet on short-term regulation of feed intake by lactating dairy cattle. J. Dairy Sci. 83:1598-1624.

Beauchemin, K. A. 2018. Invited review: Current perspectives on eating and rumination activity in dairy cows. J. Dairy Sci. 101:47624784 .

Bikker, J. P., H. van Laar, P. Rump, J. Doorenbos, K. van Meurs, G. M. Griffioen, and J. Dijkstra. 2014. Technical note: Evaluation of an ear-attached movement sensor to record cow feeding behavior and activity. J. Dairy Sci. 97:2974-2979.

Büchel, S., and A. Sundrum. 2014. Technical note: Evaluation of a new system for measuring feeding behavior of dairy cows. Comput. Electron. Agric. 108:12-16.

Grinter, L. N., M. R. Campler, and J. H. C. Costa. 2019. Technical note: Validation of a behavior-monitoring collar's precision and accuracy to measure rumination, feeding, and resting time of lactating dairy cows. J. Dairy Sci. 102:3487-3494.

Ledgerwood, D. N., C. Winckler, and C. B. Tucker. 2010. Evaluation of data loggers, sampling intervals, and editing techniques for measuring the lying behavior of dairy cattle. J. Dairy Sci. 93:5129-5139.

Mattachini, G., E. Riva, F. Perazzolo, E. Naldi, and G. Provolo. 2016. Monitoring feeding behaviour of dairy cows using accelerometers. J. Agric. Eng. 47:54-58.

Rodriguez-Jimenez, S., K. J. Haerr, E. Trevisi, J. J. Loor, F. C. Cardoso, and J. S. Osorio. 2018. Prepartal standing behavior as a parameter for early detection of postpartal subclinical ketosis associated with inflammation and liver function biomarkers in peripartal dairy cows. J. Dairy Sci. 101:8224-8235.

Schirmann, K., N. Chapinal, D. M. Weary, W. Heuwieser, and M. A. von Keyserlingk. 2011. Short-term effects of regrouping on behavior of prepartum dairy cows. J. Dairy Sci. 94:2312-2319.

van Asseldonk, M. A. P. M., A. W. Jalvingh, R. B. M. Huirne, and A. A. Dijkhuizen. 1999. Potential economic benefits from changes in management via information technology applications on Dutch dairy farms: A simulation study. Livest. Prod. Sci. 60:33-44.

Wenz, J. R. 2018. Optimization of clinical mastitis records on dairies. Vet. Clin. North Am. Food Anim. Pract. 34:493-505.

Zambelis, A., T. Wolfe, and E. Vasseur. 2019. Technical note: Validation of an ear-tag accelerometer to identify feeding and activity behaviors of tiestall-housed dairy cattle. J. Dairy Sci. 102:4536-4540.

Zehner, N., C. Umstätter, J. J. Niederhauser, and M. Schick. 2017. System specification and validation of a noseband pressure sensor for measurement of ruminating and eating behavior in stable-fed cows. Comput. Electron. Agric. 136:31-41.

\section{ORCIDS}

J. S. Osorio ๑ https://orcid.org/0000-0001-6192-0917 